\title{
A radiologically atypical case of an ampullary neuroendocrine tumor
}

\author{
Jagir Yeshwante, Maunil Bhuta, Ashwini Sankhe, \\ Jernail Singh Bava, Anagha Joshi, Tilak Dedhia
}

\begin{abstract}
Ampullary neuroendocrine tumors (ANETs) are rare tumors which show characteristic CT features. We present a case of a 42-year-old female, in which the CT features of an ampullary tumor were atypical for a neuroendocrine tumor but was confirmed on histopathology and immunohistochemistry as an NET. The uniqueness of our case was that the lesion showed mild enhancement in the arterial phase which was persistent in the venous phase with wash out in the delayed phase
\end{abstract}

Keywords: Ampullary region, Double duct sign, Neuroendocrine tumor

\section{How to cite this article}

Yeshwante J, Bhuta M, Sankhe A, Bava JS, Joshi A, Dedhia T. A radiologically atypical case of an ampullary neuroendocrine tumor. Int $\mathrm{J}$ Hepatobiliary Pancreat Dis 2015;5:17-21.

Jagir Yeshwante ${ }^{1}$, Maunil Bhuta ${ }^{1}$, Ashwini Sankhe ${ }^{2}$, Jernail Singh Bava ${ }^{3}$, Anagha Joshi ${ }^{4}$, Tilak Dedhia ${ }^{1}$

Affiliations: ${ }^{1}$ Resident, Department of Radiology, LTMMC \& LTMGH - Sion, Mumbai Maharashtra, India; ${ }^{2}$ Assistant Professor, Department of Radiology, LTMMC \& LTMGH Sion, Mumbai Maharashtra, India; ${ }^{3}$ Professor - Addl, Department of Radiology, LTMMC \& LTMGH - Sion, Mumbai, Maharashtra, India; ' Professor, Department of Radiology, LTMMC \& LTMGH - Sion, Mumbai, Maharashtra, India.

Corresponding Author: Jagir Deepak Yeshwante, Resident, Department of Radiology, LTMMC \& LTMGH - Sion, Mumbai Maharashtra, India, B-401, Ved Om Shanti CHS, Oppositejainmandir, Shanti Nagar, Dombivali (east) - 421201; Ph: 8879099870; Email: jagir.yeshwante@gmail.com

Received: 26 November 2014 Accepted: 31 January 2015

Published: 31 March 2015
Article ID: 100029IJHPDJY2015

$* * * * * * * * *$

doi:10.5348/ijhpd-2015-29-CR-4

\section{INTRODUCTION}

Neuroendocrine tumors are neoplasms that arise from cells of the endocrine and nervous systems. Many are benign, while some are malignant. These tumors are most commonly found in the small intestine, with the most common subtype being carcinoid tumors. Pancreas, lungs, etc. are other sites where these tumors are found [1].

The ultrasonography of the patient was done using a 6 Hz probe. The computed tomography (CT) scan was done on a 64 slice multi-detector CT scanner. A triple phase CT scan was done by intravenous administration of $100 \mathrm{ml}$ of contrast medium at a rate of $3 \mathrm{ml} / \mathrm{s}$ with a monophasic rate of injection. Arterial phase scanning was started after a delay of $23 \mathrm{~s}$ and portal venous scanning after a delay of $60 \mathrm{~s}$. The $1 \mathrm{~mm}$ and $5 \mathrm{~mm}$ thick sections were acquired with subsequent coronal reconstruction.

\section{CASE REPORT}

A 42-year-old female presented to the surgery OPD with complaints of weight loss with episodic pain in abdomen, yellowish discoloration of the eyes, generalized itching, vomiting since four months. On examination, mild tender hepatomegaly was noted and a clinical diagnosis of obstructive jaundice was made.

For further evaluation, an ultrasonography (USG) of the abdomen was advised, which revealed (Figure 1A):

- a small hypoechoic mass, in the peri-ampullary region with central vascularity.

- mild central and peripheral intra-hepatic biliary radicle dilatation. 
- dilatation of the Common Bile Duct and Pancreatic duct ("Double Duct Sign")

- mild hepatomegaly with a normal gallbladder.

For further evaluation and extensions of the lesion, a CT scan of the abdomen was performed which revealed (Figure 1B-D):

- a well-defined 24x23×22 mm sized, hypodense mass lesion showing mild arterial phase enhancement which persisted on venous phase in the periampullary region, projecting into the lumen of 2nd part of duodenum with no adjacent extension or invasion.

- mild central and peripheral intra-hepatic biliary radicle dilatation.

- dilatation of the common bile duct and pancreatic duct ("Double Duct Sign")

- mild hepatomegaly with a normal gallbladder.

Radiologically, after USG and CT correlation, a diagnosis of a peri-ampullary neoplasm was made. Esophagogastroduodenoscopy guided biopsy was performed.

Esophagogastroduodenoscopy revealed presence of a small sessile, fleshy mass in the peri-ampullary region jutting into the 2nd portion of duodenum (Figure 1E). A tissue biopsy was taken.

Histopathological (Figure $1 \mathrm{~F}$ ) and Immunohistochemistry report suggested the diagnosis of a low grade neuroendocrine tumor ( $\mathrm{G} 1$ - WHO classification) as the tumor cells expressed cytokeratin, synaptophysin and chromogranin.

\section{DISCUSSION}

Neuroendocrine tumors (NETs) are a large group of neoplasms with a wide spectrum of clinical, imaging, and pathologic characteristics. Imaging features of NETs can be challenging, and a multitude of imaging modalities may be required for accurate diagnosis. Accurate interpretation of the imaging findings is important to facilitate diagnosis and contribute to patient management.

Pancreatic neuroendocrine tumors, also known as NETs, are rare tumors arising from a putative common precursor, the APUD cell (amine precursor uptake and decarboxylation). These cells are of common embryological origin from the ectodermal ridge. These neuroendocrine tumors are histologically closely related to certain other neuroendocrine tumors like pheochromocytoma and medullary carcinoma of the thyroid. These tumors may or may not synthesize and secrete hormones, out of which the functioning tumors are those in which hormone secretion by the tumor results in a clinical syndrome. Non-functioning tumors are those in which either there is hormone secretion with no recognizable clinical features or no hormone secretion at all. Functioning tumors usually present early, due to the clinical symptoms, and pose a challenge for the
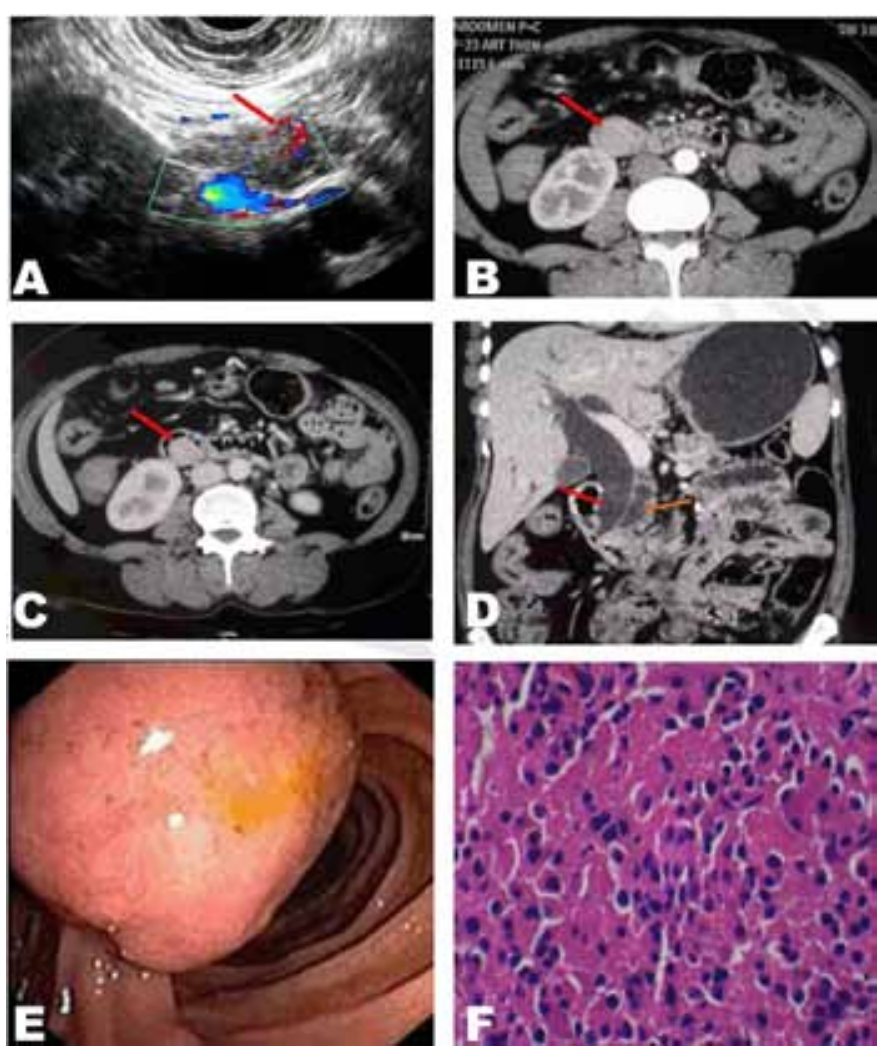

Figure 1: (A) Ultrasonography (6 $\mathrm{Hz}$ probe) showing the ampullary mass with vascularity within (red arrow), (B) Computed tomography (CT) scan (axial - arterial phase) showing mild enhancement of the ampullary mass (red arrow), (C) Computed tomography (CT) scan (axial - venous phase) showing mild enhancement of the ampullary mass (red arrow), (D) Computed tomography (CT) scan (coronalvenous phase) dilated common bile duct (red arrow) and dilated pancreatic duct (orange arrow): Double Duct Sign, (E) Esophagogastroduodenoscopy showing the ampullary mass, (F) Histopathological image of the mass lesion showing monomorphic population of small round cells.

radiologist [2].

Ampullary neuroendocrine tumor (ANET) are rare tumors, accounting for less than $1 \%$ of all gastrointestinal neuroendocrine tumors and less than $2 \%$ of all tumors of ampullary region $[2,3]$.

Histologically, although ANETs are similar to neuroendocrine tumors arising from other parts of gastrointestinal tract, they have distinct immunohistochemical features. Immunohistochemical staining is the main diagnostic method for these tumors, and ANET stain positively with chromogranin A and synaptophysin in majority of the cases [3, 4].

According to the International Classification of Diseases of Oncology, published by the World Health Organization (WHO), the ampulla has been designated a site specific code " 241 " and neuroendocrine tumors are given specific codes: neuroendocrine not otherwise specified (8246); small cell neuroendocrine carcinoma (8041); and large cell neuroendocrine carcinoma (8013) 


\section{[4].}

Radiologically, on ultrasonography, the tumor appearance is of a well-defined round mass, which is homogeneously hypoechoic in relation to the pancreas, and vascular on Doppler imaging. There may be a hyperechoic lesion or focal distortion of the pancreatic parenchyma, which may help in detecting the lesion, particularly in younger patients where the tumors tend to be less conspicuous due to the generally lower echogenicity of the pancreas. The use of enhancement pattern of these tumors using Ultrasound contrast agents, is currently under investigation. [5]

Functioning tumors are generally small, with low inherent contrast between the tumor and surrounding pancreas. Majority of islet cell tumors are hypervascular and will be best seen after intravenous injection of contrast. However, the best phase for the demonstration of these hyperattenuating small lesions is unclear [6-8]. Narrow window settings may help to improve detection. In our case, the lesion was well detected in the venous phase.

Insulinomas can be hypovascular, cystic and hypodense to the surrounding pancreas. [9]. Cystic pancreatic endocrine tumors are usually benign and non-functioning. It is difficult to differentiate them from other cystic pancreatic neoplasms on imaging alone [9]. In patients with a suspected gastrinoma, the imaging emphasis should be given to the 'gastrinoma triangle'

Large tumors are likely to be non-functioning with central necrosis and are more likely to be malignant. The features which are associated with malignancy include necrosis, large size, infiltration of the surrounding structures and calcification [10].

\section{CONCLUSION}

The uniqueness of our case was that the lesion showed mild enhancement in the arterial phase which was persistent in the venous phase with wash out in the delayed phase. These features were atypical for ANET, which was later confirmed on histopathology and immunohistochemistry.

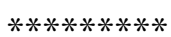

\section{Author Contributions}

Jagir Yeshwante - Substantial contributions to conception and design, Acquisition of data, Analysis and interpretation of data, Drafting the article, Revising it critically for important intellectual content, Final approval of the version to be published

Maunil Bhuta - Analysis and interpretation of data, Revising it critically for important intellectual content, Final approval of the version to be published

Ashwini Sankhe - Analysis and interpretation of data, Revising it critically for important intellectual content, Final approval of the version to be published
Jernail Singh Bava - Analysis and interpretation of data, Revising it critically for important intellectual content, Final approval of the version to be published

Anagha Joshi - Analysis and interpretation of data, Revising it critically for important intellectual content, Final approval of the version to be published

Tilak Dedhia - Acquisition of data, Drafting the article, Revising it critically for important intellectual content, Final approval of the version to be published

\section{Guarantor}

The corresponding author is the guarantor of submission.

\section{Conflict of Interest}

Authors declare no conflict of interest.

\section{Copyright}

(C) 2015 Jagir Yeshwante et al. This article is distributed under the terms of Creative Commons Attribution License which permits unrestricted use, distribution and reproduction in any medium provided the original author(s) and original publisher are properly credited. Please see the copyright policy on the journal website for more information.

\section{REFERENCES}

1. Ramage JK, Davies AH, Ardill J, et al. Guidelines for the management of gastroenteropancreatic neuroendocrine (including carcinoid) tumours. Gut 2005 Jun;54 Suppl 4:iv1-16.

2. Albores- Saavedra J, Hart A, Chable- Montero F, Henson DE. Carcinoid and high grade Neuroendocrine Carcinoma of the Ampulla of Vater- A camparitive analysis of 139 caese from Surveillance, Epidemiology, and End Results Program- A population based Study. Arch Pathol Lab Med 2010 Nov;134(11):1692-6.

3. Jaoude WA, Lau C, Sugiyama G, Duncan A. Management of Ampullary Carcinoid tumor with Pancreaticoduodenectomy. J Surg Case Rep 2010 Oct 1;2010(8):4.

4. Beasley MB, Thunnissen FB, Hasleton P. Carcinoid tumor. In Travis WD, Brambilla E, Muller-Hermelink KH. Pathology and Genetics of Tumors of the lung, Thymus, Heart. Lyon, France: IARC Press 2004:5962.

5. Oshikawa O, Tanaka S, Ioka T, Nakaizumi A, Hamada Y, Mitani T. Dynamic sonography of pancreatic tumors: Comparison with dynamic CT. AJR Am J Roentgenol 2002 May;178(5):1133-7.

6. Stafford-Johnson DB, Francis IR, Eckhauser FE, Knol JA, Chang AE. Dual-phase helical CT of nonfunctioning islet cell tumors. J Comput Assist Tomogr 1998 Mar-Apr;22(2):335-9.

7. Van Hoe L, Gryspeerdt S, Marchal G, Baert AL, Mertens L. Helical CT for the preoperative localization of islet cell tumors of the pancreas: Value of arterial and parenchymal phase images. AJR Am J Roentgenol 1995 Dec;165(6):1437-9. 
8. Chung MJ, Choi BI, Han JK, Chung JW, Han MC, Bae SH. Functioning islet cell tumor of the pancreas. Localization with dynamic spiral CT. Acta Radiol 1997 Jan;38(1):135-8.

9. Ahrendt SA, Komorowski RA, Demeure MJ, Wilson SD, Pitt HA. Cystic pancreatic neuroendocrine tumors: Is preoperative diagnosis possible? J Gastrointest Surg 2002 Jan-Feb;6(1):66-74.
10. Buetow PC, Parrino TV, Buck JL, et al. Islet cell tumors of the pancreas: Pathologic-imaging correlation among size, necrosis and cysts, calcification, malignant behavior, and functional status. AJR Am J Roentgenol 1995 Nov;165(5):1175-9.

\section{ABOUT THE AUTHORS}

Article citation: Yeshwante J, Bhuta M, Sankhe A, Bava JS, Joshi A, Dedhia T. A radiologically atypical case of an ampullary neuroendocrine tumor. Int J Hepatobiliary Pancreat Dis 2015;5:17-21.
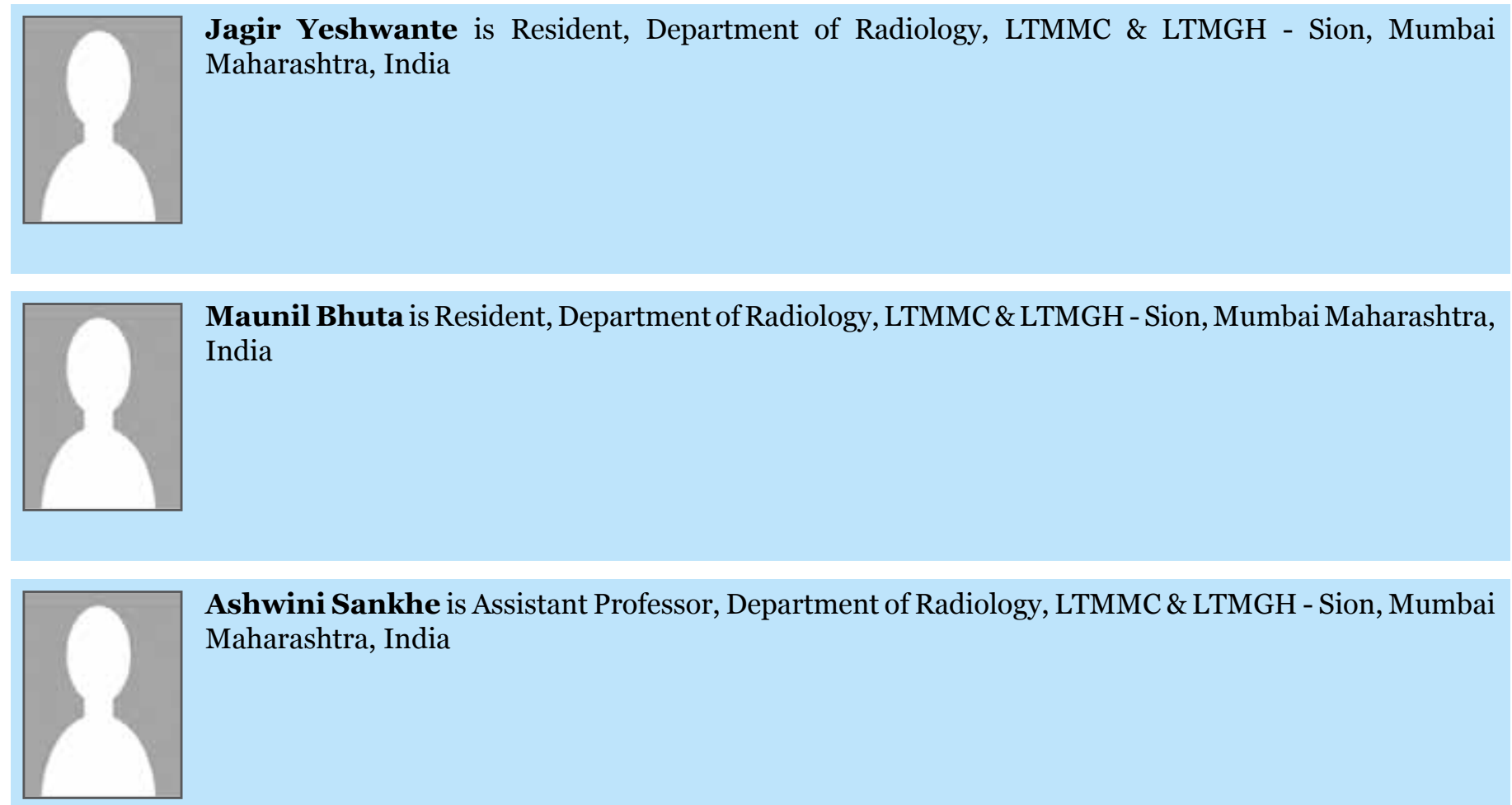

Ashwini Sankhe is Assistant Professor, Department of Radiology, LTMMC \& LTMGH - Sion, Mumbai Maharashtra, India

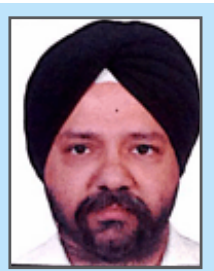

Jernail Singh Bava is Professor (Addi) at Department of Radiology at LTMMC \& GH, Mumbai, Maharashtra, India. He earned undergraduate degree MBBS and postgraduate degree of MD Radiodiagnosis from LTMMC \& GH, Mumbai, India. He has published several research papers in national and international academic journals. His research interests include hepatobiliary and pancreatic diseases and neuroradiology.

Email: jernailbava@yahoo.com

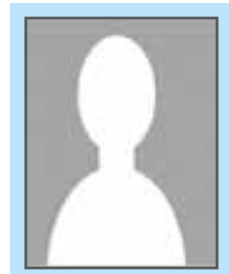

Anagha Joshi is Professor - Addl, Department of Radiology, LTMMC \& LTMGH - Sion, Mumbai, Maharashtra, India 


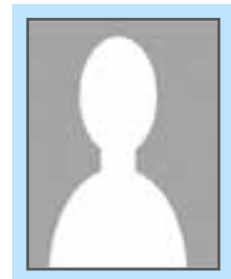

Tilak Dedhia is Resident, Department of Radiology, LTMMC \& LTMGH - Sion, Mumbai Maharashtra, India

Access full text article on other devices

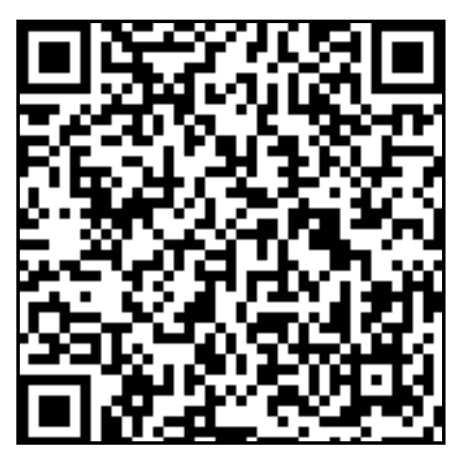

Access PDF of article on other devices

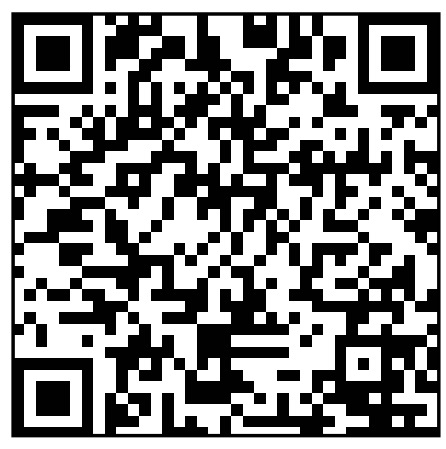

